Seceleanu Mihaela ${ }^{1}$, Ibadula Sheila ${ }^{1}$, Scrinic Olesea ${ }^{1}$, Ganta Cristina ${ }^{2}$, Circo E. ${ }^{1}$

\title{
Atherogenic index and coronarian risk - comparative assessment regarding the particularities of chronic autoimmune thyroiditis presence
}

'Department of Endocrinology, Faculty of Medicine, "Ovidius" University Constanta

${ }^{2}$ Department of Cardiology, Faculty of Medicine, "Ovidius" University Constanta

\begin{abstract}
Objectives: Assessment of autoimmune cause hypothyroidism and dyslipidemia involvement in the apparition of major vascular complications. Methods: A total of 152 patients were investigated appreciating in comparison to a healthy control lot the hormone serum level, the presence of antimicrosomal thyroid antibodies and the serum levels of lipids. Atherogenic index and coronarian risk were calculated and correlated with the incidence of coronarian and cerebral vascular accidents. Results: Among the patients with goiter it was noted a high incidence of a subclinical hypothyroidism $(31,58 \%)$. Thyroid autoimmunity was involved in $94,4 \%$ of the patients with clinical hypothyroidism, in $93,7 \%$ with subclinical hypothyroidism and $100 \%$ in the patients with thyrotoxicosis. Low serum level of HDL-cholesterol was identified in $66,6 \%$ of patients with clinical hypothyroidism and $64,5 \%$ patients with subclinical hypothyroidism. The assessment of atherogenic index and coronarian risk was significantly higher $(p<0,01)$ in patients with hypothyroidism in comparison to healthy control subjects. The incidence of vascular accidents was significantly higher $(\mathrm{p}<0,01)$ among the hypothyroid patients $(19,7 \% / 10,8 \%)$, of masculine gender $(12,7 \%)$ where the main cause of Eduard Circo
\end{abstract}

Fagetului str. 163 bis, code: 900654 , Constanta, Romania, phone: + 40241512251;

email: eduard_circo@yahoo.com; hypothyroidism was autoimmunity. Conclusions: The atherogenic index and coronarian risk were higher in patients with hypothyroidism associated to thyroid autoimmunity resulting in an increased probability in producing vascular accidents.

Keywords: hypothyroidism, dyslipidemia, atherogenic index, coronarian risk, antimicrosomal thyroid antibodies.

\section{Introduction}

Thyroid functional impairment presents an important metabolic risk with major cardiovascular impact. $[1 ; 2 ; 3 ; 4 ; 5 ; 6]$ Associating thyroid autoimmunity amplifies this risk.[3; 7]

Vascular impairment becomes an additional effect for thyroid hormone deficit and even if still unclear, for the presence of thyroid autoantibodies, known as "aggressive" on brain microcirculation, which created the concept of Hashimoto's "encephalitis". 
Table II: Patients with goiter

\begin{tabular}{|c|c|c|c|}
\hline & $\begin{array}{c}\mathbf{1 9 - 3 0} \\
\text { years }\end{array}$ & $\begin{array}{c}\mathbf{3 1}-\mathbf{4 5} \\
\text { years }\end{array}$ & $\begin{array}{c}\mathbf{4 6}-\mathbf{5 3} \\
\text { years }\end{array}$ \\
\hline $\mathbf{F}(\mathbf{n}=\mathbf{8 8})$ & $21(13,8 \%)$ & $35(25 \%)$ & $32(21 \%)$ \\
\hline$M(\mathbf{n}=\mathbf{6 4})$ & $12(7,9 \%)$ & $27(17,7 \%)$ & $25(16,4 \%)$ \\
\hline & $33(21,7 \%)$ & $62(40,7 \%)$ & $57(37,4 \%)$ \\
\hline
\end{tabular}

with goiter (OMS classification) and a control group of 252 healthy persons in terms of thyroid function, without known personal or family history regarding thyroid pathology.

The parameters studied were:

1. Ultrasound: thyroid volume and echogenicity

2. Evaluation of thyroid function (ECL): TSH, Free-T4, Free-T3.

3. Lipidic profile: HDL-cholesterol, LDLcholesterol, triglycerides (TG)

4. TPOAb serum level (ECL) defined pathological as a value $>35 \mathrm{UI} / \mathrm{L}$.

5. The incidence of vascular cerebral and/ or coronary accidents based on medical documents presented by patient.

6. Atherogenic index prediction (AI) (LDL-c/ HDL-c report), considered normal if $<3.5$; the value of this index was considered to influence the coronary risk (CR) being inversely correlated with serum HDLcholesterol level (Table I).

Table I- Coronary risk (FRIEDWALD) [2]

\begin{tabular}{|c|c|c|c|c|}
\hline \multirow{3}{*}{$\begin{array}{c}\text { The } \\
\text { Coronarian } \\
\text { Risk } \\
\end{array}$} & \multicolumn{2}{|c|}{ Females } & \multicolumn{2}{|c|}{ Males } \\
\hline & \multicolumn{4}{|c|}{ HDL - cholesterol } \\
\hline & g / I & $\mathrm{mmol} / \mathrm{l}$ & $\mathrm{g} / \mathrm{l}$ & $\mathrm{mmol} / \mathrm{l}$ \\
\hline 0,5 & 0,70 & 1,81 & 0,60 & 1,55 \\
\hline 1,0 & 0,55 & 1,42 & 0,45 & 1,16 \\
\hline 1,5 & 0,45 & 1,16 & 0,35 & 0,90 \\
\hline 2,0 & 0,35 & 0,90 & 0,25 & 0,64 \\
\hline$>2$ & $<0,35$ & $<0,90$ & $<0,25$ & $<0,64$ \\
\hline
\end{tabular}

\section{Results and discussions}

The structure of the two study groups according to their gender and age was as follows (Table II and Table III).

Thyroid echogenicity was appreciated as being normal/low. In subjects with thyroid hypoechogeny serum levels of antimicrosomal antibodies (TPOAb) were dosed (Table V).

Table $V$ - : Incidence of chronic autoimmune thyroiditis in patients with thyroid hypoechogeny

\begin{tabular}{||c|c|c||}
\hline & $\begin{array}{c}\text { Negative } \\
\text { TPOAb }\end{array}$ & $\begin{array}{c}\text { Pathologic } \\
\text { TPOAb }\end{array}$ \\
\hline $\mathbf{F ~ ( n = 5 0 )}$ & $18(18,6 \%)$ & $32(33 \%)$ \\
\hline $\mathbf{M}(\mathbf{n}=\mathbf{4 7})$ & $23(23,7 \%)$ & $24(24,7 \%)$ \\
\hline & $41(42,3 \%)$ & $56(57,7 \%)$ \\
\hline
\end{tabular}

The obtained data reveals an increased incidence of chronic autoimmune thyroiditis in females compared to men. However, it is noted that values of TPOAb considered non-pathological in patients with hypoecogenic thyroid were significantly ( $p<0,01)$ more frequent $(64,7 \% / 32,6 \%)$ among patients selected according to ultrasound criteria. Under functional aspect the patients were investigated 
by dosage of serum level of TSH, FT4 and FT3 (Table VI).

Table VI - Thyroid function

\begin{tabular}{|c|c|c|c|c|}
\cline { 2 - 5 } \multicolumn{1}{c|}{} & Normal & $\begin{array}{c}\text { Clinical } \\
\text { hypothyroidism }\end{array}$ & $\begin{array}{c}\text { Subclinical } \\
\text { hypothyroidism }\end{array}$ & Thyrotoxicosis \\
\hline $\begin{array}{c}\text { Study group } \\
(\mathbf{n}=\mathbf{1 5 2})\end{array}$ & $84(55,26 \%)$ & $18(11,8 \%)$ & $48(31,5 \%)$ & $2(1,3 \%)$ \\
\hline $\begin{array}{c}\text { Control group } \\
(\mathbf{n}=\mathbf{2 5 2})\end{array}$ & $240(95,23 \%)$ & 0 & $12(4,76 \%)$ & 0 \\
\hline
\end{tabular}

Among the patients with goiter associating thyroid functional disorders was found a high incidence of subclinical hypothyroidism $[\mathrm{n}=48(31,58 \%)]$. Patients with thyroid functional disorders $(n=68)$ were characterized under the aspect of registered serum level of TPOAb (Table VII).

Table VII: Autoimmunity involvement in achieving thyroid functional disorders

\begin{tabular}{|l|c|c|}
\hline Patients & $\begin{array}{c}\text { Pathologic } \\
\text { TPOAb (n) }\end{array}$ & $\begin{array}{c}\text { Negative } \\
\text { TPOAb (n) }\end{array}$ \\
\hline $\begin{array}{l}\text { Clinical } \\
\text { hypothyroidism } \\
\text { (n= 18) }\end{array}$ & $\begin{array}{l}17 \\
\text { Subclinical }\end{array}$ & $\begin{array}{c}1 \\
\text { Sub }\end{array}$ \\
$\begin{array}{l}\text { hypothyroidism } \\
\text { (n= 48) }\end{array}$ & $45,7 \%$ & 3 \\
\hline $\begin{array}{l}\text { Thyrotoxicosis } \\
(\mathbf{n = 2})\end{array}$ & 2 & $6,2 \%$ \\
\hline
\end{tabular}

Subjects in control group presenting a hormonal profile characteristic to subclinical hypothyroidism (SCHT) showed the following characteristics (Table VIII)

Table VIII: Patients with subclinical hypothyroidismWitness lot

\begin{tabular}{|l|c|c|c|c|c|}
\hline & $\begin{array}{c}\text { Pathologic } \\
\text { TPOAb } \\
\text { (n) }\end{array}$ & $\begin{array}{c}\text { Negative } \\
\text { TPOAb } \\
\text { (n) }\end{array}$ & $\begin{array}{c}\text { AGE } \\
\text { (years) }\end{array}$ & \multicolumn{2}{|c|}{ Gender } \\
\cline { 4 - 6 } & 9 & 3 & $49 \pm 4$ & 12 & M \\
\hline $\begin{array}{l}\text { Patients } \\
\text { SCHT }\end{array}$ & & & & & \\
\hline
\end{tabular}

SCHT patients presenting pathological values of serum TPOAb level were considered having "asymptomatic" chronic autoimmune thyroiditis, but with evolutionary potential.

Lipid profile was achieved measuring the following constants: HDL-colesterol, LDL- colesterol and TG. Significant pathological differences were found according to hormonal changes (Table IX).

\begin{tabular}{|c|c|c|c|c|c|c|c|c|c|c|}
\hline & \multirow{2}{*}{$\begin{array}{l}\text { Thyroid } \\
\text { function }\end{array}$} & \multicolumn{3}{|c|}{ HDL cholesterol } & \multicolumn{3}{|c|}{ LDL cholesterol } & \multicolumn{3}{|c|}{ Triglicerins } \\
\hline & & $\uparrow$ & $\mathbf{N}$ & $\downarrow$ & $\uparrow$ & $\mathbf{N}$ & $\downarrow$ & $\uparrow$ & $\mathbf{N}$ & $\downarrow$ \\
\hline \multirow{5}{*}{$\begin{array}{l}\text { Study } \\
\text { group } \\
n=152\end{array}$} & Normal $(n=84)$ & 11 & 30 & 43 & 46 & 30 & 8 & 41 & 35 & 8 \\
\hline & $\begin{array}{c}\text { Subclinical } \\
\text { hypothyroidism } \\
(\mathrm{n}=48)\end{array}$ & 2 & 15 & 31 & 29 & 16 & 3 & 25 & 20 & 3 \\
\hline & $\begin{array}{c}\text { Clinically } \\
\text { manifested } \\
\text { hypothyroidism } \\
(\mathrm{n}=18)\end{array}$ & 2 & 4 & 12 & 15 & 3 & - & 14 & 3 & 1 \\
\hline & $\begin{array}{l}\text { Thyrotoxicosis } \\
(\mathrm{n}=2)\end{array}$ & - & - & 2 & - & - & 2 & - & - & 2 \\
\hline & $\%$ & 9.8 & 32.2 & 57.8 & 59.2 & 32.2 & 8.5 & 52.6 & 38.1 & 9.2 \\
\hline
\end{tabular}

Considering a low serum level of HDLcholesterol in order of calculating the vascular risk, this parameter was rated separately (Table X).

Table X. - Patients with low serum HDL-colesterol

\begin{tabular}{|c|c|c|c|}
\hline & Thyroid function & HDL $\downarrow$ & $\%$ \\
\hline \multirow{3}{*}{$\begin{array}{c}\text { Study } \\
\text { group } \\
(\mathrm{n}=152)\end{array}$} & Normal $(n=84)$ & 43 & $51,1 \%$ \\
\hline & $\begin{array}{l}\text { Subclinical hypothyroidism } \\
\qquad(\mathrm{n}=48)\end{array}$ & 31 & $64,5 \%$ \\
\hline & $\begin{array}{c}\text { Clinically manifested } \\
\text { hypothyroidism }(\mathrm{n}=18)\end{array}$ & 12 & $66,6 \%$ \\
\hline
\end{tabular}

The percentage of low serum levels prevailed among hypothyroid patients.

Rating atherogenic index (AI) and coronary risk (CR) found pathological values distribution by the type of thyroid functional disorders (Table XI).

Table XI- Correlation of IA/IRC/ thyroid functional status

\begin{tabular}{|c|c|c|c|c|c|c|}
\cline { 2 - 7 } \multicolumn{1}{c|}{} & \multicolumn{3}{c|}{ AI } & \multicolumn{3}{c|}{ CR } \\
\hline $\begin{array}{c}\text { Thyroidian } \\
\text { function }\end{array}$ & $\begin{array}{c}\text { Study } \\
\text { group }\end{array}$ & $\begin{array}{c}\text { Control } \\
\text { group }\end{array}$ & $\mathbf{p}$ & $\begin{array}{c}\text { Study } \\
\text { group }\end{array}$ & $\begin{array}{c}\text { Control } \\
\text { group }\end{array}$ & $\mathbf{p}$ \\
\hline Normal & 3.22 & 2.68 & $<0.01$ & 2.18 & 1.23 & $<0.01$ \\
\hline $\begin{array}{c}\text { Clinical } \\
\text { hypothyroidism }\end{array}$ & 4.84 & & & & & \\
\hline $\begin{array}{c}\text { Subclinical } \\
\text { hypothyroidism }\end{array}$ & 4.73 & 3.87 & $<0.01$ & 3.42 & 2.67 & $<0.01$ \\
\hline Tyrotoxicosis & 2.4 & & & 1.3 & & \\
\hline
\end{tabular}

Overall cardiovascular risk is markedly increased in patients with goiter compared to the control group $(p<0,01)$. Statistical significance is maintained among patients with subclinical hypothyroidism, for those with goiter existing additional risks linked to the period and intensity of thyroid autoimmunity process evolution. 
The incidence of stroke and coronary accidents was different in the two study groups (Table XII).

\begin{tabular}{|c|c|c|c|c|c|c|c|c|c|c|c|}
\hline & \multirow{2}{*}{$\begin{array}{c}\text { Nr. of } \\
\text { vascular } \\
\text { accid. }\end{array}$} & \multicolumn{2}{|c|}{$\begin{array}{c}19-30 \\
\text { years }\end{array}$} & \multicolumn{2}{|c|}{$\begin{array}{c}31-45 \\
\text { years }\end{array}$} & \multicolumn{2}{|c|}{$\begin{array}{c}46-53 \\
\text { years }\end{array}$} & \multicolumn{2}{|c|}{ M } & \multicolumn{2}{|c|}{$F$} \\
\hline & & $\mathrm{M}$ & $F$ & $\mathrm{M}$ & $F$ & $\mathrm{M}$ & $\mathrm{F}$ & \multirow{3}{*}{$\begin{array}{l}\text { Study } \\
\text { group }\end{array}$} & \multirow{3}{*}{$\begin{array}{c}\text { Control } \\
\text { group }\end{array}$} & \multirow{3}{*}{$\begin{array}{l}\text { Study } \\
\text { group }\end{array}$} & \multirow{3}{*}{$\begin{array}{c}\text { Control } \\
\text { group }\end{array}$} \\
\hline $\begin{array}{l}\text { Study } \\
\text { group }\end{array}$ & $\begin{array}{c}19 \\
(12.5 \%)\end{array}$ & 1 & - & 3 & 2 & 8 & 5 & & & & \\
\hline $\begin{array}{c}\text { Control } \\
\text { group }\end{array}$ & $\begin{array}{c}13 \\
(5.16 \%) \\
\end{array}$ & 1 & - & 2 & 1 & 6 & 3 & & & & \\
\hline & & & & & & & & $7.9 \%$ & $3.5 \%$ & $4.6 \%$ & $1.6 \%$ \\
\hline
\end{tabular}

Findings for each study group by gender, age and thyroid functional level, regarding the incidence of strokes registered significant differences (Table XIII).

A high incidence of vascular accidents was found in men from the maximum age group and also among hypothyroid patients $(\mathrm{p}<0,01)$.

The association of hypothyroidism with thyroid autoantibodies represents a potential additional risk factor in the development of degenerative vascular pathology.

\section{Conclusions}

1. Autoimmune thyropathy is associated frequently to thyroid functional disorders.

2. The presence of hypothyroidism increases the vascular risk and the atherogenic index.
3. The increased frequency of strokes is priorly found in hypothyroid patients group with present thyroid autoantibodies.

4. Early identification of potential hypothyroidism ("sub-subclinical") characterized only by the presence of thyroid autoantibodies, of subclinical and patent hypothyroidism represents an activity of preventive care.

\section{References}

1. Ichiki, T. (2010). Thyroid hormone and atherosclerosis. Vascul Pharmacol, 52(3-4), 151156. doi: 10.1016/j.vph.2009.09.004

2. Singh, S., Duggal, J., Molnar, J., Maldonado, F., Barsano, C. P., \& Arora, R. (2008). Impact of subclinical thyroid disorders on coronary heart disease, cardiovascular and all-cause mortality: a meta-analysis. Int J Cardiol, 125(1), 41-48. doi: 10.1016/j.ijcard.2007.02.027

3. Ciccone, M.M., De Pergola, G., Porcelli, M. T., Scicchitano, P., Caldarola, P., Iacoviello, M. \& Favale, S. (2010). Increased carotid IMT in overweight and obese women affected by Hashimoto's thyroiditis: an adiposity and autoimmune linkage? BMC Cardiovasc Disord, 10, 22. doi: 10.1186/1471-2261-10-22

4. Toruner, F., Altinova, A. E., Karakoc, A., Yetkin,

Table XIII - The incidence of strokes in patients with normothyroidism (NT) and hypothyroidism (HT) correlated to their gender and age.

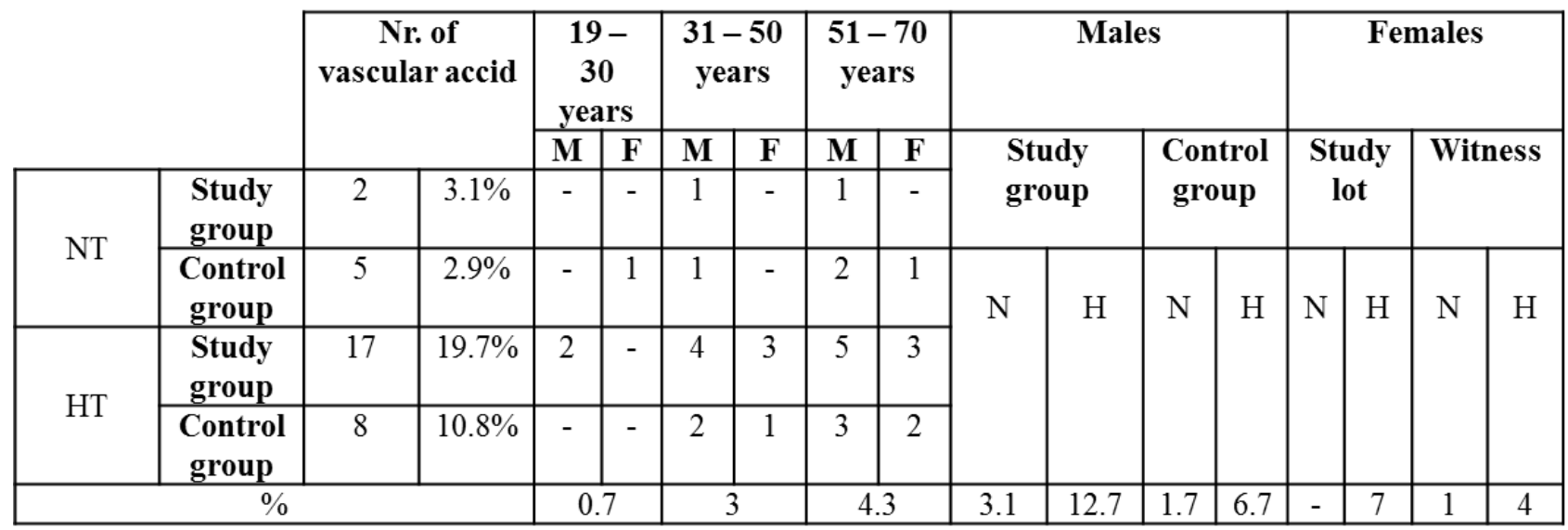


I., Ayvaz, G., Cakir, N. \& Arslan, M. (2008). Risk factors for cardiovascular disease in patients with subclinical hypothyroidism. Adv Ther, 25(5), 430-437. doi: 10.1007/s12325-008-0053-7

5. Valentina, V. N., Marijan, B., Chedo, D. \& Branka, K. (2011). Subclinical hypothyroidism and risk to carotid atherosclerosis. Arq Bras Endocrinol Metabol, 55(7), 475-480.

6. Palmieri, E. A., Fazio, S., Lombardi, G. \& Biondi, B. (2004). Subclinical hypothyroidism and cardiovascular risk: a reason to treat? Treat
Endocrinol, 3(4), 233-244.

7. Thvilum, M., Brandt, F., Brix, T. H. \& Hegedus, L. (2012). A review of the evidence for and against increased mortality in hypothyroidism. Nat Rev Endocrinol, 8(7), 417-424. doi: 10.1038/ nrendo.2012.29 